\title{
Prévention des situations à risque de forte charge émotionnelle : exemple dans le secteur de l'aide à domicile
}

\section{Prevention of high emotional labor risky situations: an example in home help sector}

\author{
Corinne Van De Weerdt, Olivier Morel, Catherine Caël* \\ * INRS, Département Homme au Travail, Rue du Morvan, CS 60027, 54519 Vandœuvre Lès Nancy Cedex, \\ France, tél : 0383508790, mail : corinne.vandeweerdt@inrs.fr
}

\section{Introduction}

Dans le monde du travail actuel, les risques psychosociaux (RPS) sont encore inquiétants. Ce contexte amène les entreprises à se pencher de plus en plus sur les émotions au travail. Celles-ci sont explorées pour tenter de saisir quelles en sont les causes, comment elles émergent, quel rôle elles jouent sur les comportements, ou encore, comment elles interviennent dans la régulation des situations difficiles. Cette attractivité est relativement nouvelle car les émotions au travail ont pendant longtemps été ignorées, ou pire, considérées comme des perturbateurs de la pensée rationnelle. Aujourd'hui, leur prise en compte est investie, car elle semble prometteuse en termes de moyen de compréhension des mécanismes en œuvre et de prévention des situations à risques sur le plan psychosocial.

Dans les secteurs d'activité professionnelle où la relation avec des personnes extérieures à l'entreprise est centrale, les émotions au travail sont particulièrement examinées. Les attentes envers les salariés de ce secteur peuvent être grandes de la part des organisations. La qualité de service étant de mise, les émotions exprimées au public comportent une valeur fondamentale. Cela peut créer des exigences émotionnelles fortes pour les salariés, qui se voient soumis à des pressions et des objectifs élevés sur le plan des émotions.

On peut alors se demander si cette situation n'engendre pas une charge émotionnelle qui peut être à risque pour les salariés, au niveau de leur motivation, leur engagement au travail, leur santé. En effet, si les émotions manifestées par les salariés font l'objet d'une attention particulière dans les entreprises, cela ne provoque-t-il pas une augmentation des exigences au travail ? Face à cela, est-il possible de mettre en place des moyens de prévention?

Pour apporter des éléments de réponse à ces questions, un cadre théorique a été mobilisé et une recherche-intervention a été menée. Il a été question d'étudier les émotions au travail en lien avec la santé, pour saisir les exigences au travail, essentiellement émotionnelles, et prévenir les situations à risque. L'objet a été d'identifier, dans une entreprise d'aide à domicile, les situations à forte charge émotionnelle auxquelles les salariés sont confrontés. La recherche-intervention a consisté à examiner les régulations individuelles et collectives des émotions en situation réelle et à développer une méthodologie d'intervention centrée sur les émotions, afin d'agir sur la transformation du travail en faveur de la prévention.

\section{Cadre théorique}

Les risques psychosociaux (RPS) sont loin de disparaître dans le monde du travail. Ils correspondent à des situations de travail où sont présents, combinés ou non : du stress au travail, des violences internes et externes, mais aussi, du mal-être, de la souffrance, de l'inquiétude, etc. Selon la dernière enquête de la Fondation Européenne de Dublin de 2015 (Eurofound, 2016), 25\% de salariés interrogés déclarent être touchés par le stress. La France n'est pas épargnée par ce risque, comme le montre la dernière enquête nationale sur les conditions de travail (Dares, 2014). L'exposition des salariés aux RPS peut avoir des conséquences sur leur santé. Les RPS peuvent être induits par l'activité elle-même ou par l'organisation et les relations de travail (Cox, 1993 ; Cox et al., 2005).

A l'heure où les risques psychosociaux sont préoccupants, les émotions au travail sont de plus en plus examinées. Parce qu'elles peuvent être notamment le reflet d'une souffrance au travail, ou au contraire, participer activement à la régulation des difficultés rencontrées, elles font de plus en plus souvent l'objet d'une 
attention particulière dans les organisations. En interaction étroite avec la cognition, elles jouent un rôle prépondérant dans le travail. Depuis une vingtaine d'années, on sait que les émotions sont nécessaires à la cognition. Elles interviennent dans la mémoire (Ledoux, 1994 ; Cahill et al., 1996), la prise de décision (Damasio, 1994, 1999) ou encore la prise de risques (Isen, 1993). Au sens large, les émotions correspondent à « tous les événements ou états du champ affectif qui se caractérisent par un ensemble d'éprouvés psychiques spécifiques accompagnés, de façon variable en intensité et en qualité, de manifestations physiologiques et comportementales » (Cosnier, 1994, p.14).

Pourtant, elles n'ont pas toujours occupé une place de choix. Considérées pendant longtemps comme « illogiques », « subjectives », « irrationnelles », « incontrôlables », les émotions étaient surtout étudiées comme des variables intermédiaires à contrôler. Dans les travaux anglo-saxons notamment (Tversky \& Kahneman, 1974, 1981 ; Izard, 1977 ; Ajzen \& Kruglanski, 1983 ; Lerner \& Tiedens, 2006), les émotions étaient souvent étudiées en tant que biais interférant avec la cognition.

Les émotions au travail sont reconnues aujourd'hui comme des agents à la fois bénéfiques à la réalisation du travail et aux performances, et régulateurs de situations difficiles ou contraignantes (Grosjean \& Ribert-Van De Weerdt, 2005, 2010). Elles se définissent comme «des états affectifs qui ne sont pas à considérer comme un facteur marginal intervenant de temps en temps, mais bien comme constitutifs de toute activité qui se déploie, dans un flot constant et mouvant d'états émotionnels, qui orientent et impactent l'action, la relation et la réflexion. Ces états émotionnels sont eux-mêmes modifiés par l'activité déployée et le sens que les personnes construisent de la situation» (Cahour \& Van De Weerdt, 2016). Parmi les conséquences d'une émotion, on trouve par exemple l'expression émotionnelle, l'orientation sur l'objet et la tendance d'action (par exemple l'approche, l'évitement), l'interruption ou la modification du comportement actuel.

Le travail peut néanmoins susciter une charge émotionnelle, qui se définit comme «le fardeau, le poids des états subjectifs intenses que vit une personne étant donné ses rôles au travail, ses missions, les choses ou les personnes dont elle a la responsabilité » (Hellemans, 2014, p.90).

Il existe deux types de charge émotionnelle : celle provenant de la nature du travail, et celle issue de la prescription (Ribert-Van De Weerdt \& Baratta, 2016). Les salariés ont tendance à considérer, lorsque la charge émotionnelle provient essentiellement de la nature du travail, que cette charge fait partie du métier, et qu'il est de leur responsabilité de la gérer, et le plus souvent, seul (Loriol, 2012). Or, des actions collectives de prévention peuvent être mises en place pour ces deux types de charge émotionnelle, apportant aux salariés des effets bénéfiques et protecteurs vis-à-vis de la santé (Ribert-Van De Weerdt \& Baratta, 2016).

En outre, la littérature scientifique montre que la charge émotionnelle est très souvent liée à la présence d'interactions avec d'autres personnes, dans des situations demandant une perception des émotions des autres et la maîtrise des siennes pour mener à bien son travail. C'est le cas lorsqu'il y a la présence de tensions avec le public ou les collègues, d'isolement ou de manque d'entraide dans le travail (Gollac \& Bodier, 2010), d'échanges interpersonnels chargés d'émotions (Lachance, 2006), de situations d'agressivité physique et verbale, de contact avec des personnes en détresse, de tension avec la hiérarchie, les collègues, le public (Klein \& Long, 2013). Plus rarement, les travaux scientifiques montrent que la charge émotionnelle est liée aux conditions de travail perçues comme défavorables (Cahour, 2010; Cahour \& Lancry, 2011), indépendamment de relations interpersonnelles. Or, certains salariés utilisent leur propre émotivité pour travailler, y compris pour des activités manuelles, isolées, à la chaîne, ne nécessitant pas d'interactions verbales, mais générant des sources de confort ou d'inconfort émotionnel au travail selon les moments. La charge émotionnelle peut donc émaner d'interactions interpersonnelles autant que de prescriptions. Cette charge peut provenir de réactions émotionnelles au travail, de la gestion de ses propres émotions, ou de celle des personnes à qui on est confronté, ou bien encore à des objectifs quantitatifs trop élevés, à l'intensification du travail, etc.

On distingue par ailleurs deux natures différentes de la charge émotionnelle: spécifique et globale. Comme le précise Hellemans (2014), la charge globale renvoie «aux réactions émotionnelles liées à un ensemble de conditions de travail perçues comme non idéales ou défavorables », alors que la charge spécifique « découle de la nature même du travail inscrite dans les relations interpersonnelles » [65, p.90].

Si l'on considère d'une part les deux types de charge émotionnelle et d'autre part ses deux natures, on peut donc définir la charge émotionnelle spécifique comme « la charge issue de contraintes de travail situées perçues comme non idéales ou défavorables, émanant de situations de nature relationnelle ou non, connotée négativement, de forte intensité émotionnelle ou de faible intensité mais à fréquence élevée (Cahour \& Van De Weerdt, 2016 ; Ribert-Van De Weerdt \& Baratta, 2016).

En lien avec la notion de charge émotionnelle, ce que l'on appelle «exigences émotionnelles » 
constituent des conditions d'exposition des salariés à des situations qui créent des contraintes sur le plan affectif. Ces exigences correspondent à la nécessité de «maîtriser et façonner » ses propres émotions, afin notamment de «maîtriser et façonner » celles ressenties par les personnes avec qui on interagit lors du travail, comme le spécifient Gollac et Bodier (2010). Le contrôle et la maîtrise des émotions constituent un vrai travail en soi. Il s'agit de ce que Hochschild (1983) appelle «le travail émotionnel ». Ce travail nécessite des efforts pour être accompli, comme le précise Mann (2004). Il engendre des conséquences, que Soares (2002) qualifie de "prix » (2002) et Scherer (2006) de " poids ». Quel que soit le terme utilisé, l'idée est que le travail émotionnel constitue une exigence émotionnelle. Les exigences émotionnelles ont quatre principaux déterminants que Gollac et Bodier distinguent : la relation au public, le contact avec la souffrance, le fait de devoir cacher ses émotions, et la peur au travail (par exemple de l'accident, des violences externes, de l'échec).

Dans le secteur tertiaire, où la valeur ajoutée tient à la qualité de service, les émotions des salariés et des clients sont d'autant plus « scrutées » qu'elles sont estimées comme centrales (Ashforth \& Humphrey, 1993 ; Briner, 1999, Soares, 2002). Les salariés peuvent donc être confrontés à de fortes exigences émotionnelles (Hochschild, 1983). Ils doivent fréquemment réaliser un «travail émotionnel », intimement lié à la part relationnelle de l'activité, exigeant la perception des émotions des autres et la maîtrise des siennes (Totterdell \& Holman, 2003 ; Krauth-Gruber, 2009 ; Ribert-Van De Weerdt, 2011).

Si l'on considère le cas particulier de salariés travaillant au domicile de clients pour leur apporter une aide ou un soin, ce secteur fait l'objet d'une attention grandissante (David, Cloutier, Teiger \& Prévost, 2000 ; Van de Weerdt, 2011). Les raisons sont liées à des enjeux multiples qui sont à la fois politiques, économiques, sociaux, juridiques, de santé publique et au travail (Baillieul, Chaillot \& Benoteau, 2013; Dares, 2015 ; Garabige, 2015). Près de trois millions de salariés travaillent dans l'aide et le soin à domicile (pour des activités de garde d'enfant, ménage, aide aux activités de la vie courante, soins). Néanmoins, ce domaine connait des besoins encore croissants, liés notamment à l'évolution des caractéristiques générales de la population (élévation de la durée de vie, préférence pour le maintien à domicile, etc.), la politique sociale actuelle (volonté de maîtrise des coûts d'hospitalisation, de professionnalisation, etc.), et au manque de durabilité dans l'emploi (en 2013, on relevait $35 \%$ de turn-over et $25 \%$ d'absentéisme). Ce dernier aspect est lié au caractère peu attractif du secteur et à sa sinistralité élevée (Dares, 2014). La liste des risques professionnels exposant ces salariés est longue (chimiques, biologiques, infectieux, électriques, routiers, de troubles musculo-squelettiques, d'accidents de plain-pied, psychosociaux, etc.). Sur le plan des risques psychosociaux, figurent en bonne place le stress et les violences externes, causées par des déterminants techniques et organisationnels propres au secteur. Comme nous le verrons dans l'étude de cas présentée, un grand nombre de déterminants agissent en interaction, créant ainsi une accumulation de contraintes de travail que les salariés doivent gérer, et des exigences au travail multiples. Parmi elles, figurent les exigences émotionnelles, qui peuvent engendrer une forte charge émotionnelle pour les salariés, courante dans ce secteur (Cloutier, David, Teiger, C. \& Prévost, 1999 ; Gagnon et al., 2003).

Ainsi, il a été question d'examiner, au moyen d'une recherche-intervention, les conditions de réalisation de l'activité par des professionnels de ce secteur, pour saisir les exigences du travail, y compris émotionnelles, afin de prévenir les situations à risque. De même, il s'agissait d'identifier les situations à forte charge émotionnelle auxquelles les salariés peuvent être confrontés. L'enjeu se situe tant au niveau de la qualité de service (Cloutier, David \& Teiger, 2003) que de la prévention des risques professionnels.

\section{Problématique et hypothèses}

Cette recherche-intervention de type psycho-ergonomique avait pour finalité d'étudier les émotions au travail en lien avec la santé. Dans un objectif de prévention, et plus précisément, vis-à-vis des situations à risque de forte charge émotionnelle, il était question d'examiner les exigences du travail, essentiellement de nature émotionnelle, et leurs effets sur l'activité. Le secteur retenu est celui de l'aide à domicile. La régulation des émotions par les salariés de ce secteur se situe au centre des analyses, pour saisir la complexité des tâches, les conditions de réalisation de l'activité, les déterminants de l'activité et des émotions, et les compétences mobilisées. Sur un plan plus empirique, il était question d'examiner les régulations individuelles et collectives des émotions en situation réelle, et d'agir sur la transformation du travail et la prévention. Les objectifs opérationnels ont consisté en :

- la construction d'une méthodologie spécifique

- l'application de cette méthodologie sur le terrain dont la particularité est de concerner des métiers en mutation (professionnalisation, nouveaux diplômes, etc.) et des activités dont une part importante concerne 
la régulation des émotions.

Pour répondre aux objectifs fixés, des hypothèses ont été avancées :

1. les situations à forte charge émotionnelle créent des exigences émotionnelles pouvant entraîner des effets à terme sur la santé et la motivation des salariés ;

2. la charge émotionnelle spécifique, relative au travail situé, a des effets à plus long terme sur la charge émotionnelle globale, par l'accumulation de situations vécues alourdissant la charge globale ;

3. en agissant sur les conditions de travail, il est possible de faciliter la régulation des émotions des salariés exposés à des situations à forte charge émotionnelle, ou à des situations à charge émotionnelle plus modérée mais de façon répétée.

\section{Terrain et méthodologie}

Le terrain de la recherche-intervention est une PME du Grand Est, employant 34 personnes, créée il y a 11 ans, qui s'est développée très rapidement $(+11 \%$ de croissance en $2013 ;+18,3 \%$ de croissance en 2014). Ses domaines d'activité sont : l'aide à domicile, l'entretien de la maison, le portage de repas, la téléassistance, l'accompagnement en véhicule. Elle gère 180 clients pour l'aide à domicile et 13 clients pour le portage de repas. L'objet de la demande était de comprendre pourquoi les taux de turn-over (de 29\%) et d'absentéisme (de $22 \%$ ) étaient si élevés.

Les analyses ont porté sur l'activité et les émotions au travail des intervenants à domicile en identifiant les déterminants, les régulations cognitives et émotionnelles et les effets sur la santé perçue. Les caractéristiques du personnel sont comparables aux statistiques nationales du secteur :

- la moyenne d'âge est de 41 ans (45 ans pour le secteur),

- $60 \%$ sont sans diplôme ou sans rapport avec l'activité exercée,

- l'expérience professionnelle est pour 70\% des personnes sans rapport avec l'activité précédente,

- l'ancienneté moyenne dans l'entreprise est de 3 ans.

Le recueil de données sur le terrain a eu lieu au moyen d'entretiens, d'observations, de qualifications subjectives des situations de travail par les salariés, et d'auto-confrontations collectives (Tableau 1).

Tableau 1 : Récapitulatif synthétique de la méthode d'intervention.

\begin{tabular}{|c|c|c|c|}
\hline & $\begin{array}{c}\text { Prise de connaissance de la } \\
\text { structure }\end{array}$ & $\begin{array}{l}\text { Analyse de la perception des } \\
\text { contraintes, des risques et } \\
\text { des effets sur la santé }\end{array}$ & Analyse de l'activité \\
\hline$\frac{\sqrt[n]{e}}{\frac{0}{0}}$ & $\begin{array}{l}\text {-'organisation interne, les } \\
\text { métiers, la nature des } \\
\text { tâches, les modes de } \\
\text { communication interne, la } \\
\text { culture du secteur et } \\
\text { d'entreprise, le contexte } \\
\text { socio-économique, etc. }\end{array}$ & $\begin{array}{l}\text { - } \quad \text { contraintes de travail } \\
\text { ressenties } \\
\text { - santé perçue en lien avec le } \\
\text { travail }\end{array}$ & $\begin{array}{l}\text { - } \quad \text { activité en situation réelle } \\
\text { d'activité }\end{array}$ \\
\hline 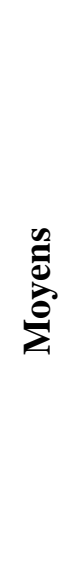 & $\begin{array}{l}\text { - } \quad \text { analyses de documents } \\
\text { - } \quad \mathbf{~ e n t r e t i e n s ~ a ̀ ~ d i f f e ́ r e n t s ~} \\
\text { niveaux de l'entreprise }\end{array}$ & 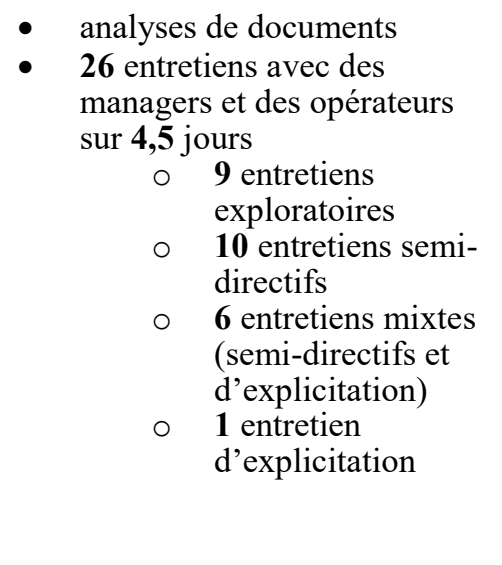 & 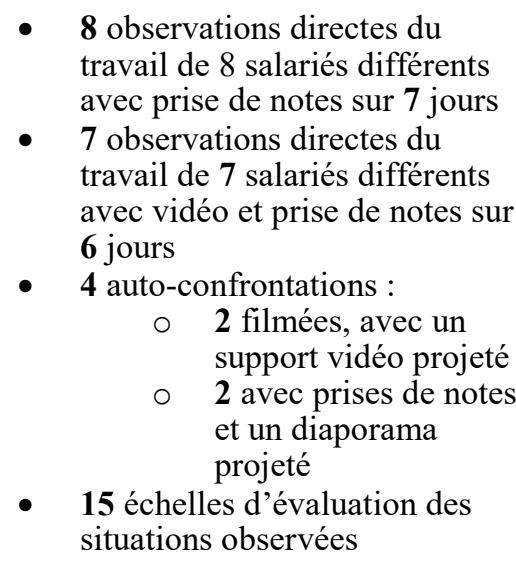 \\
\hline
\end{tabular}


La phase de prise de connaissance de la structure a consisté à analyser des documents internes à l'entreprise (par exemple, le référentiel des métiers utilisé, les données chiffrées sur la croissance de l'entreprise, sa situation économique et sociale, etc.). Puis des entretiens de quatre types différents ont été réalisés (exploratoires, semi-directifs, d'explicitation et mixtes) et enregistrés par dictaphone. Les entretiens semidirectifs avaient pour but d'approfondir les thèmes à explorer, les entretiens d'explicitation ou mixtes visaient à focaliser sur les thèmes examinés et/ou à faire expliciter un événement émotionnellement marquant choisi par le sujet.

Ensuite, des observations ont été effectuées. Elles ont consisté à suivre sur le terrain le travail de 15 salariés et à identifier par ce moyen plusieurs situations sensibles ou difficultés rencontrées en situation réelle. Un enregistrement vidéo a été utilisé lorsque l'accord des personnes concernées avait été donné (dans le cas contraire, des notes ont été prises). A la fin de chaque séance d'observation, des échelles de Likert en 7 points ont été remplies par l'observateur sur la base de l'évaluation faite par les salariés observés, sur quatre dimensions, qui sont : les niveaux d'intensité de la charge de travail, de difficulté des cas à traiter, de fatigue ressentie, et de qualification de la journée en termes de bonne ou mauvaise journée (toutes ces dimensions pouvant être nuancées sur une échelle graduée). De plus, une question ouverte a permis de récolter en fin d'observation des commentaires supplémentaires sur cette journée de travail passée. Les séquences vidéo obtenues ont été analysées et sélectionnées à des fins d'utilisation lors des auto-confrontations (ainsi que les notes prises présentées sous forme de diaporama).

Deux auto-confrontations filmées ont utilisé un support vidéo (réunissant trois salariés pour l'une, puis deux salariés pour l'autre; deux auto-confrontations ont été effectuées avec prises de notes et un diaporama projeté (rassemblant trois salariés pour l'une puis deux salariés pour l'autre). Ces auto-confrontations collectives ont été menées avec deux finalités : (a) comprendre l'activité et les émotions au travail (en présentant des images signifiantes, en suscitant des commentaires, en analysant les situations de travail qui posent question, en les confrontant pour connaître leur niveau de généralité, en mettant en lumière les stratégies et les compromis en œuvre) ; (b) transformer le travail (en créant un cadre permettant l'expression des différentes dimensions du vécu au travail de type émotionnel, en utilisant la vidéo comme objet médiateur de co-construction de la pensée et de la parole sur le travail, en favorisant la pensée réflexive, en faisant débattre des conditions de travail, en envisageant collectivement leur changement).

Les débats ont amené à formaliser des pistes concrètes de solutions pour faire face aux difficultés rencontrées. L'avancée de propositions pratiques a été discutée au sein des groupes directement concernés puis validées par ces derniers, pour aboutir à une liste de propositions applicables et co-construites.

\section{Résultats}

Les résultats issus des analyses de terrain sont organisés en quatre parties. La première présente les déterminants techniques et organisationnels de l'activité et des émotions. La deuxième expose les tâches, activités et émotions ressenties au travail. La troisième porte sur la régulation émotionnelle et les conditions qui l'entravent. Enfin, la quatrième présente le plan d'actions.

\subsection{Les déterminants techniques et organisationnels}

Les déterminants techniques et organisationnels de l'activité et des émotions sont à la fois variés et nombreux. Leur nombre accumulé crée des exigences au travail élevées, y compris sur le plan émotionnel, comme le montrent les extraits d'entretien et d'observation présentés :

- l'environnement physique et technique : le lieu de travail est discret, isolé, intime et privé

(Extrait d'entretien : "quand vous ne connaissez pas la maladie de Parkinson, et que vous voyez une dame par terre en train de faire une crise, vous ne savez pas quoi faire, ce n'est pas évident. Parce que ni l'infirmière peut être là, personne ne peut être là, il faut qu'elle ait une piqûre, mais quoi faire? Comment faire? Nous on n'a pas le droit, strictement pas le droit, on ne sait même pas qui avertir. »)

- l'organisation du travail : les décisions concernant la prestation sont prises par des personnes externes, les contraintes dans le travail sont fortes au niveau organisationnel et temporel 
(Extrait d'entretien: "quand on a qu'une demi-heure pour faire une toilette, une dame avec un déambulateur qui met déjà quinze minutes pour aller à la salle de bains, je ne vais quand même pas la pousser pour aller plus vite dans mon planning!»)

- les relations de travail : les échanges formels entre collègues sont rares, la violence verbale externe existe

(Extrait d'entretien : "On ne se croise jamais, on se fait la bise vite fait quand on se croise dans le bureau; je n'en connais pas la moitié de mes collègues, je pense qu'une réunion une fois par an ce $n$ 'est pas suffisant, on a tellement de choses à se raconter, une fois qu'on tombe en formation ça part dans tous les sens ; on n'arrive pas à vider notre sac. »)

(Extrait d'observation: "La cliente panique, elle ne trouve plus son alliance, elle cherche partout, elle accuse l'assistante de vie, elle crie, elle parle vulgairement, elle invective pendant 3 minutes et 40 secondes, jusqu'au moment où l'alliance est retrouvée sur le tapis par l'assistante de vie. »)

- la tâche et le contenu du travail à effectuer : les prestations ont lieu auprès d'un public varié, les frontières entre les missions sont floues, les responsabilités élevées et les exigences émotionnelles fortes

(Extrait d'entretien : «je voudrais une formation pour m'apprendre à dire non, ou savoir comment réagir à des mots grossiers. ».

Extrait d'entretien : "On a un monsieur qui est très difficile, et souvent il fait de grosses crises de colère ; au début j'étais paniquée ; maintenant j'essaie de faire avec, mais bon, le problème persiste et il n'y a rien qui change; il est suivi psychologiquement, il a des cachets à prendre mais il ne les prend pas, je ne peux rien faire, à part appuyer auprès de sa tutrice, mais sinon, je ne vois pas. ».

- l'environnement macro-économique : les moyens financiers dans le secteur sont bas, la situation économique du secteur est incertaine

(Extrait d'entretien : "Quand je vois que mon salaire est en dessous du seuil de pauvreté, j'ai mal au cour. »).

\subsection{Les tâches, activités et émotions ressenties par les salariés}

En théorie, les tâches diffèrent selon les différents types de métiers des intervenants à domicile (d'après le référentiel interne à l'entreprise, basé sur une nomenclature des métiers). Mais les analyses de l'activité montrent que toutes les prestations peuvent être attribuées à tous les intervenants, indifféremment de leur qualification. Les aides-ménagers ont pour mission théorique «d'accomplir, chez des personnes âgées, un travail matériel, moral et social, contribuant au maintien à domicile». Les assistants de vie devraient exclusivement « aider à la réalisation de tâches domestiques et administratives pour personnes âgées ou handicapées ». Les auxiliaires de vie ont des tâches dédiées «à aider des personnes fragiles à assumer seules les activités quotidiennes pour un maintien à domicile en autonomie », et sont formés «à intervenir dans des contextes spécifiques dits «difficiles». L'analyse de l'activité montre que les salariés interviennent dans toutes les situations qui se présentent, car inscrites dans leur programme de manière indifférenciée, exerçant à tour de rôle :

- auprès du même public (des personnes âgées, malades ou handicapées),

- pour des missions identiques (l'entretien du logement par du ménage et du repassage, l'achat des courses, la préparation des repas, l'aide aux activités quotidiennes (la toilette, l'alimentation, la mobilité), la stimulation sociale et relationnelle (discuter avec le bénéficiaire, faire le lien avec la famille), la coordination avec l'équipe soignante, la participation à l'évaluation (des conditions matérielles, de la santé de la personne), l'adaptation à la situation présente (fin de vie, hospitalisation à domicile, pathologies spécifiques, troubles psychiques, etc.).

Les chroniques d'activité révèlent des glissements de tâches, des attributions de missions dépassant les rôles, des prises de responsabilité hors cadre et un décalage entre leurs compétences acquises et celles mises en œuvre. La figure 1 et ses données chiffrées montrent des écarts importants entre les tâches et les activités des assistants et auxiliaires de vie. Par exemple, l'activité comporte plus de ménage que prévu théoriquement dans les tâches. Au contraire, la part consacrée à la stimulation sociale est trop réduite par rapport ce qu'elle devrait représenter. 
Figure 1: La réparation des tâches et des activités des assistants et auxiliaires de vie.

Tâches

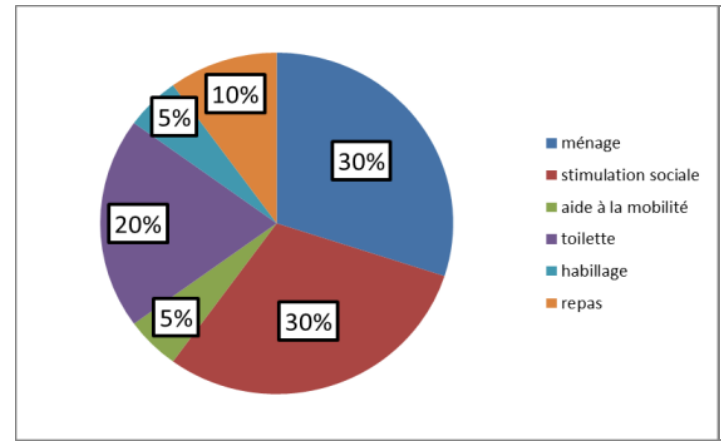

Activités

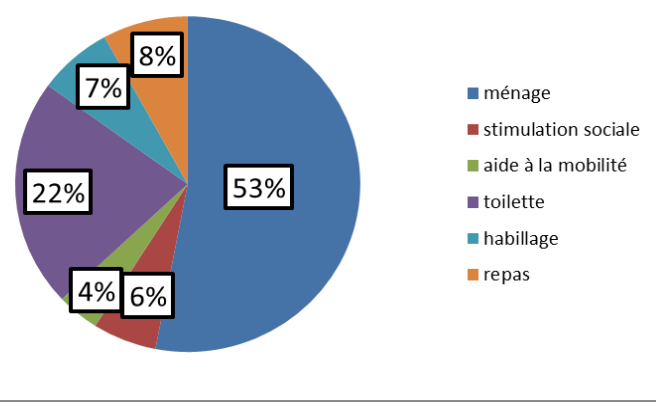

Les entretiens et les verbalisations en observations et en auto-confrontation ont permis de mettre en évidence que ces écarts entre les tâches prescrites et l'activité créent des émotions négatives chez les intervenants à domicile, qui considèrent que les compétences ne sont pas employées à bon escient, et les amènent à questionner leur rôle et le sens de leur travail : "J'ai trop de ménages par rapport à ma qualification. »; "On discute avec le client, ça fait partie de notre travail, et après on nettoie une pièce, on ressort et on rediscute, parce qu'on ne va pas lui dire qu'on n'a pas le temps. On fait des allers et retours pour faire les deux en même temps. »; "Ce n'est pas donné à tout le monde de savoir prendre la personne, elle peut s'énerver très vite, je ne suis pas qualifiée, j'apprends sur le tas. Mais des fois ce n'est pas facile. Je me dis que je ne devrais pas le faire. C'est trop risqué. ».

Les résultats des évaluations réalisées auprès des salariés sur différentes dimensions du travail au moyen des échelles de Likert, montrent une certaine cohérence interindividuelle dans la manière dont les salariés perçoivent leur activité sur le plan émotionnel (Graphe 1). L'échelle va de 1 à 7 (1 étant le niveau bas de la dimension évaluée et 7 le niveau le plus élevé).

Pour la grande majorité des intervenants à domicile, la journée reste satisfaisante car estimée par eux comme étant une «bonne journée », malgré la charge élevée et une forte fatigue ressentie. Une journée pour eux peut être chargée et fatigante mais sans forcément être perçue comme mauvaise pour autant. La question ouverte posée en plus des réponses aux échelles, relative à la nature des déterminants associés à ces évaluations, a révélé que le principal critère pour estimer une journée comme «bonne » est lié au caractère agréable des bénéficiaires. Une journée difficile ou chargée n'est donc pas évaluée forcément comme mauvaise. Cela n'affecte pas la journée d'une tonalité émotionnelle négative, contrairement à la relation avec le bénéficiaire.

Graphe 1 : Evaluation des journées observées par les intervenants à domicile.

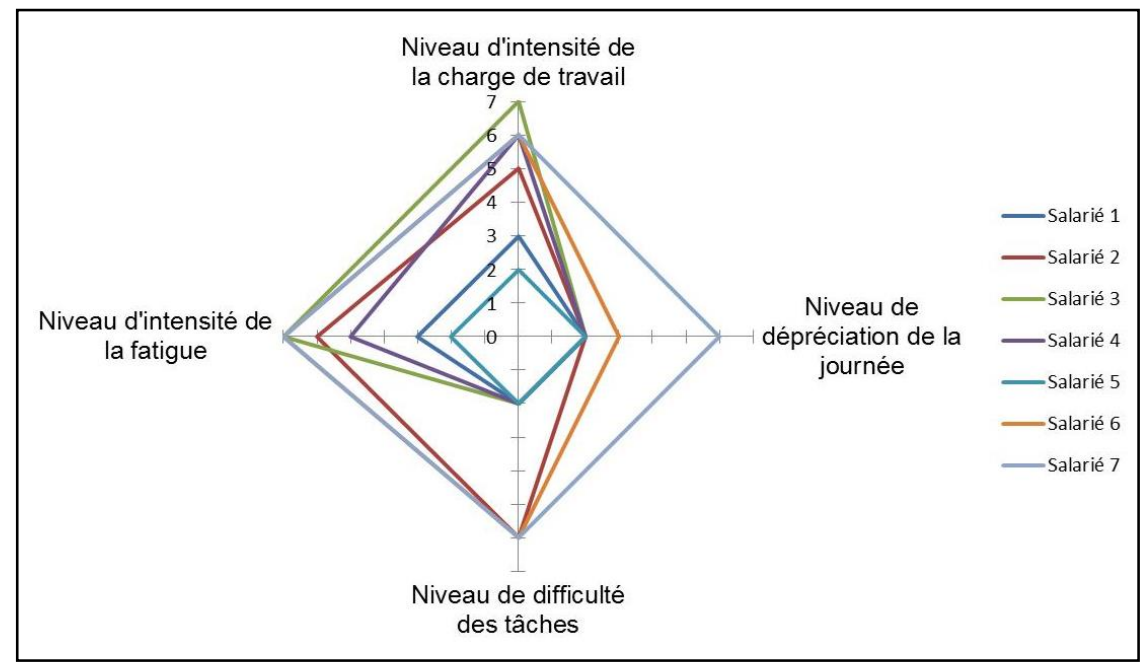




\subsection{La régulation émotionnelle}

La régulation émotionnelle s'inscrit dans un processus dynamique complexe, que nous représentons dans la figure 2. S'inspirant de la théorie de Zapf (2008), elle met en lumière une dynamique favorisant une régulation de l'activité et des émotions de nature fonctionnelle sauf en cas de conflits non débattus. Elle présente les conditions dans lesquelles la régulation de l'activité et des émotions serait optimale s'il n'y avait pas la présence de freins engendrés par ces conflits (Figures 2 et 3 ).

Figure 2 : Dynamique schématique d'une régulation fonctionnelle de l'activité et des émotions (adaptation du modèle de Zapf, 2008).

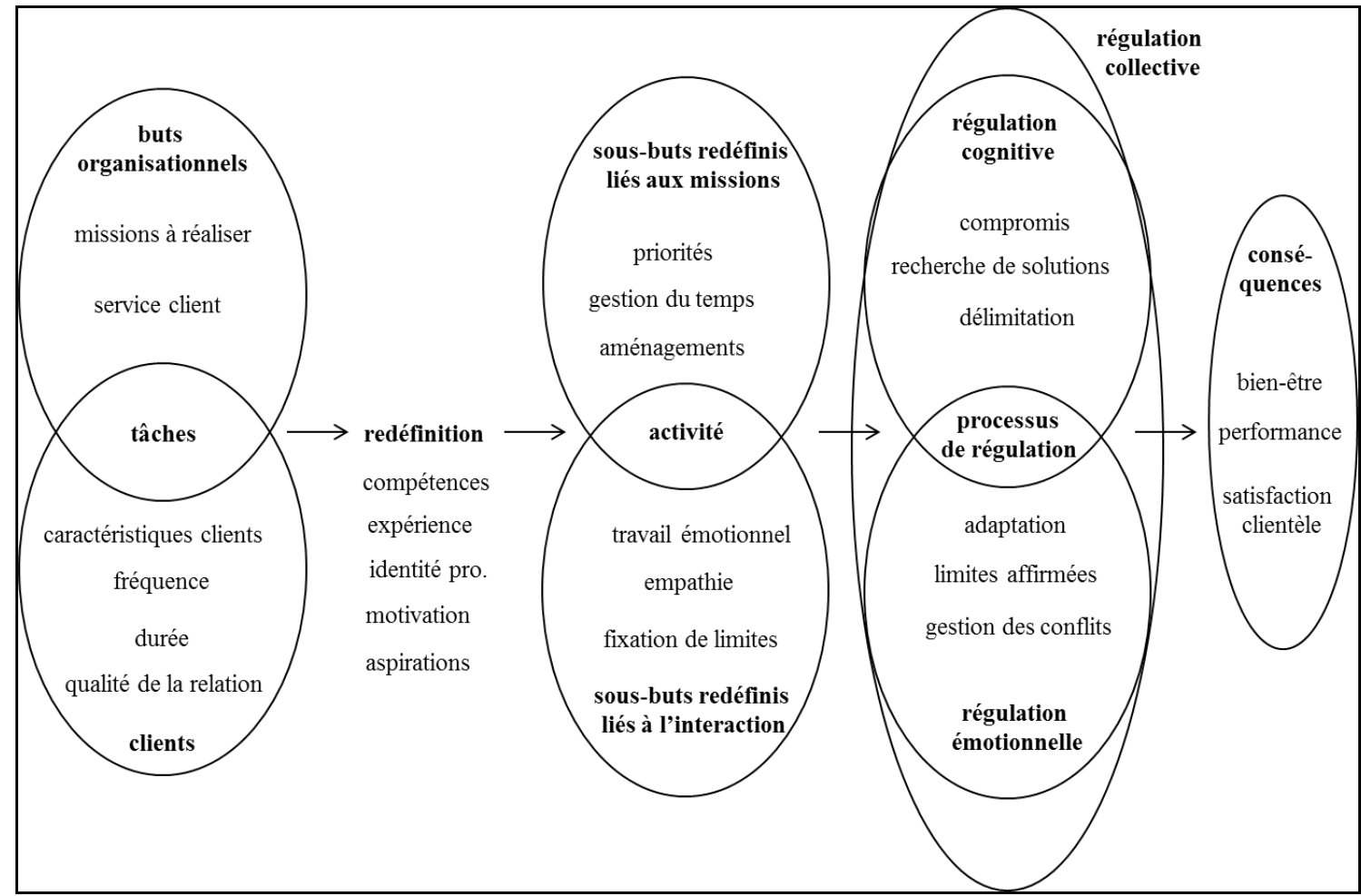

Cette figure 2 se lit de la gauche vers la droite. Elle fait apparaittre en haut l'ensemble des buts organisationnels (comprenant les missions à réaliser et le service client à assurer), et en bas, l'ensemble représenté par les clients ainsi que leurs caractéristiques. L'intersection correspond aux tâches. Pour passer des tâches à l'activité, une redéfinition des tâches s'opère. Elle dépend des compétences, de l'expérience, de l'identité professionnelle, de la motivation et des aspirations des salariés. Ceci amène à des sous-buts qui sont redéfinis, relativement aux missions, et aux interactions avec les clients. Les exigences du travail concernent l'ensemble des buts et des sous-buts redéfinis. Les exigences émotionnelles sont celles qui se réfèrent aux interactions. L'activité est réalisée en fonction de ces sous-buts redéfinis. Les conditions de sa réalisation nécessitent pour les salariés de procéder à des régulations, à la fois cognitives et émotionnelles. Il s'agit d'un processus qui repose, si l'on considère le plan cognitif, sur des compromis, la recherche de solutions aux problèmes rencontrés et une délimitation des rôles. Au niveau émotionnel, les régulations visent l'adaptation aux situations et aux clients, mais aussi à réaffirmer les limites et à gérer les conflits interpersonnels. La régulation de l'activité et des émotions a des conséquences sur l'efficacité, en termes de performance et de qualité de service rendue aux clients, mais aussi sur le bien-être.

Les données issues des entretiens, des observations et des auto-confrontations révèlent que les compétences des intervenants à domicile sont fortement sollicitées. Elles sont basées sur des connaissances acquises en grande partie sur l'expérience, y compris pour les compétences émotionnelles. Celles-ci ont trait aux dimensions sociales, relationnelles, communicationnelles, mais aussi de gestion de l'incertitude, de persuasion, etc. (Leduc \& Valléry, 2006). Ces compétences, qui permettent d'identifier, comprendre, exprimer, utiliser ses propres émotions et celles des autres, jouent un rôle essentiel dans la performance au travail et dans les relations 
sociales. Nos données confirment ce que les travaux de Leduc et Valléry (2010) avaient montré. Les savoir mobilisés touchent aux : savoir, savoir-faire, savoir-être, savoir «ne pas dire », savoir se contenir, savoir réagir, savoir différer sa réaction, etc. Les aptitudes appliquées concernent directement : la réactivité, la résistance (sur le plan physique et moral), l'empathie, le sens des responsabilités, l'affirmation de soi, etc.

Nos observations montrent que les intervenants à domicile mettent en œuvre des compétences émotionnelles pour gérer les différentes situations de travail, en maîtrisant leurs émotions, et la plupart du temps, seuls. Or, comme l'a montré Rimé (2005), le partage des émotions avec autrui constitue « un besoin insatiable », surtout lorsqu'il s'agit du vécu d'événements émotionnels majeurs. Si des conflits apparaissent au niveau de la redéfinition des buts, et que l'intervenant reste seul avec ces conflits sans pouvoir en parler, des effets négatifs peuvent survenir sur sa motivation et sa santé. La figure 3, basée sur la figure 2, illustre comment des conflits non débattus en sein de l'entreprise peuvent amener à freiner le processus de régulation, entraînant une surcharge cognitive et émotionnelle, et à plus long terme, des effets néfastes sur la santé et le bien-être (souffrance au travail, burnout, etc.). Nos résultats issus des entretiens et des observations en situation réelle de travail mettent en évidence que la régulation des émotions des salariés est entravée par des conditions de travail néfastes, en raison de déterminants techniques et organisationnels agissant comme des freins.

Figure 3 : Dynamique schématique d'une régulation dysfonctionnelle de l'activité et des émotions (adaptation du modèle de Zapf, 2008).

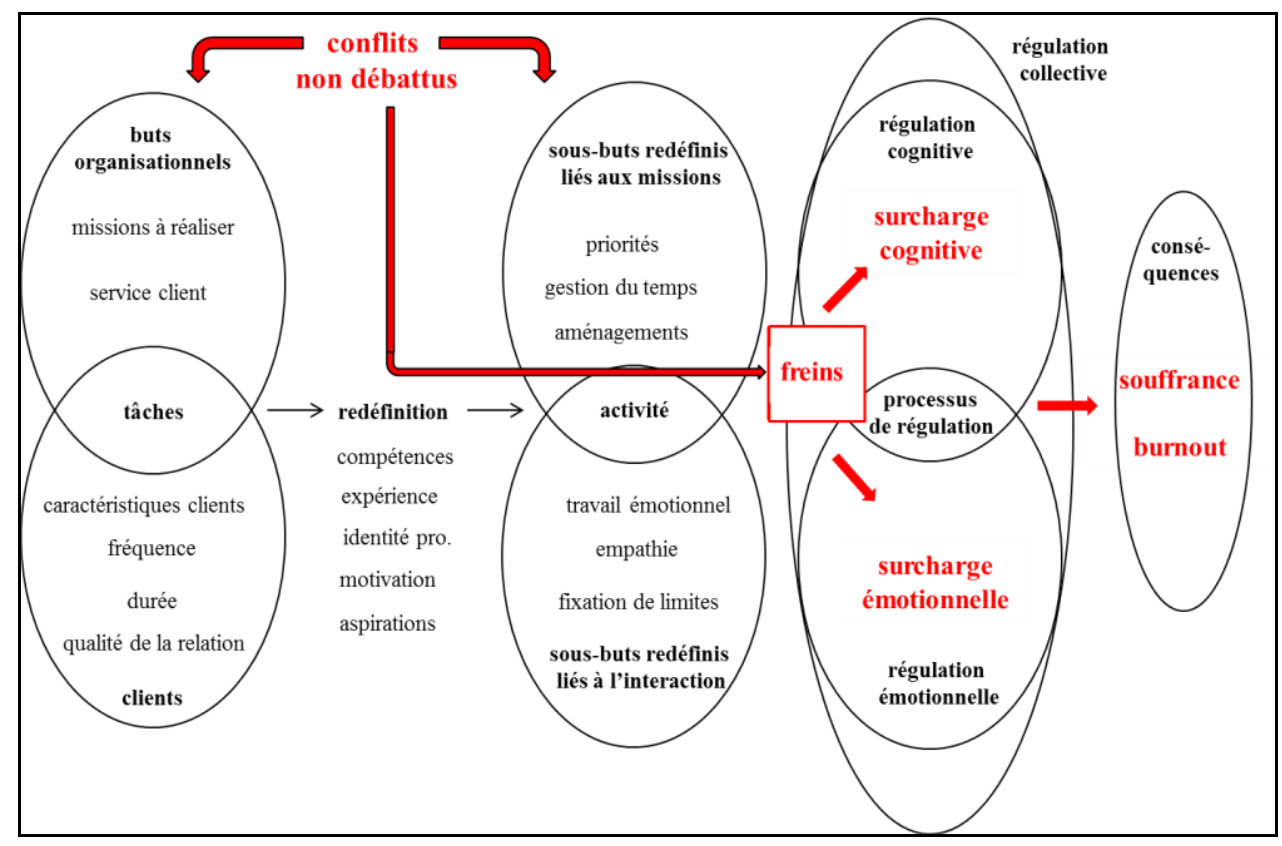

Ces extraits d'entretiens et de verbalisations en observation l'illustrent : «Je fais trop de ménages par rapport à ma qualification, ça me démotive complètement.» "Je viens de recevoir mon planning, j'ai une personne que je ne connais pas, je ne sais pas ce qu'il faut faire, il va falloir que je me débrouille, c'est lourd. » "Quand vous êtes amenée à être insultée, je vous avoue que moi qu'il m'est arrivé de me mettre en colère, et pourtant c'est une chose que je n'ai pas envie. » «Je veux bien y aller, chez ce client, pour aider, mais au bout d'un moment, c'est bon quoi, je n'en peux plus, on n'est pas là non plus pour se faire insulter. » «J'étais dans un état où je me disais "je n'y retourne pas, je m'en vais, je vais voir ailleurs. » Mais bon, je ne pouvais pas faire ça du jour au lendemain. C'est une situation qui n'aurait pas été correcte. " Je suis moins motivée, parfois les matins je me dis "pourquoi tu y vas? ", mais après je me dis, "il faut avancer, il faut le faire. 》 «Je ne peux rien décharger, tout ce que j'absorbe de ce que j'entends, et tout ça engendre un stress. » "J'ai des problèmes de cervicales, et je fais des malaises régulièrement, des crises de spasmophilie, de plus en plus souvent; dans mon sac à main, je ne sais pas combien il y a de cachets, mais je ne les prends pas, sinon je ne vais pas finir ma journée! Les gens sont durs avec nous, mais on ne peut pas les abandonner comme ça. On résiste, mais jusqu'à quand?».

Ainsi, la régulation de l'activité et des émotions joue donc un rôle sur la santé des personnes, mais aussi 
sur leur engagement professionnel (ou désengagement selon les cas) (Figures 2 et 3). Dans ce secteur, la dimension relationnelle constitue un élément constitutif fondamental de motivation et d'engagement au travail. Lorsque les intervenants parviennent à trouver des relations de qualité avec les clients, cela participe à les engager davantage.

En outre, l'identité professionnelle se construit sur l'image sociale du métier, mais aussi sur les représentations personnelles, alimentées par les connaissances et l'expérience, et par la constitution d'une «posture professionnelle» fondée sur des règles. L'identité et les règles professionnelles sont construites en groupe. Cela suppose l'existence d'un collectif de travail, qui se reconnaît comme tel (Faucheux \& Moscovici, 1971). Selon Rocher et ses collaborateurs (2005), un métier est d'autant plus porteur de reconnaissance et protecteur de la santé et de l'usure des salariés qu'il a défini des règles de métier, c'est-à-dire un ensemble de points de repères communs pour l'exercice quotidien du métier. Dans le secteur de l'aide à domicile, ces règles font souvent défaut. Les résultats ont montré que les intervenants à domicile ont bâti des règles personnelles dans la solitude. L'absence de règles de métier partagées et discutées peut entraver la réalisation même du travail au quotidien. Il peut créer des difficultés d'ordre relationnel et de suivi des principes individuels en cas de divergence de pratiques avec d'autres collègues. Des formations adaptées, outre l'apport de connaissances, peuvent également servir à renforcer l'identité professionnelle et les règles de métier.

\subsection{Le plan d'actions comprenant les mesures spécifiques et celles généralisables}

L'identification des déterminants techniques et organisationnels constitue un enjeu de santé et d'efficacité pour les salariés. En agissant sur ces déterminants, en termes de prévention, il est possible de :

- permettre aux individus de faire un travail « de qualité »,

- rendre possibles les régulations de l'activité et des émotions,

- maintenir une charge émotionnelle à un niveau acceptable,

- permettre aux individus et aux collectifs d'agir sur le travail et ses déterminants.

En augmentant les possibilités de régulation, par la mise à disposition de ressources - de nature individuelle (et subjective), collective (et discutée), et organisationnelle (et partagée) - il est possible de favoriser les régulations tout en limitant les efforts fournis en prévenant l'usure physique et psychologique. Ceci permet aussi de renforcer l'identité professionnelle, construire ou réaffirmer les règles de métier, apporter un soutien aux salariés (dans tous les sens du terme), la reconnaissance de leurs compétences, et de rompre la solitude.

Les pistes de solution ont été élaborées en collaboration avec l'entreprise. A partir des données d'entretiens, d'observation et d'auto-confrontation recueillies, une pré-liste de recommandations a été préparée, pointant les aspects du travail à faire évoluer (en priorité) en vue de l'amélioration des conditions de travail, et ceux à maintenir dans la durée. Durant deux séances (intermédiaire et finale) de restitution des résultats, les recommandations ont été discutées, amendées et adaptées au mieux pour faciliter leur application. Ces séances ont réuni la direction, le responsable de secteur et les délégués du personnel.

Les déterminants de l'activité et des émotions qu'il convenait de faire évoluer pour atteindre de meilleures conditions de travail portaient sur cinq domaines: la préparation des missions, le suivi des prestations, la formation, l'accueil des nouveaux embauchés, le repérage des situations à risques.

Le plan d'actions relate les aspects matériels, managériaux, émotionnels et organisationnels. Le tableau 3 présente un extrait de ce plan, se focalisant sur les actions qui portent uniquement sur les aspects émotionnels du travail. Il fait figurer des pistes spécifiques à l'entreprise étudiée mais qui sont généralisables à d'autres entreprises du domaine de l'aide à la personne, voire à d'autres secteurs d'activité.

Certaines pistes ont déjà pu être mises en œuvre dans l'entreprise et d'autres sont en cours de développement. La validation des principales actions présentées a pu avoir lieu lors de la restitution finale des résultats. La liste des pistes d'action, si elle est très riche, reste néanmoins non exhaustive étant donné les nombreuses solutions qui pourraient encore être apportées. Cette liste a pour ambition de constituer une base pour aider l'entreprise à aménager les conditions de travail, ainsi qu'un outil de dialogue entre les membres de l'entreprise pour la constitution collective des modifications organisationnelles. Cette présentation sous forme tabulaire met en relation des problèmes et des solutions, de façon apparemment unilatérale et déterministe, pour en faciliter la lecture. Dans la réalité, des recouvrements existent, une solution pouvant être rattachée à différents problèmes, et un problème pouvant trouver différentes solutions. 
Tableau 3 : Extrait du plan d'action portant sur les aspects émotionnels.

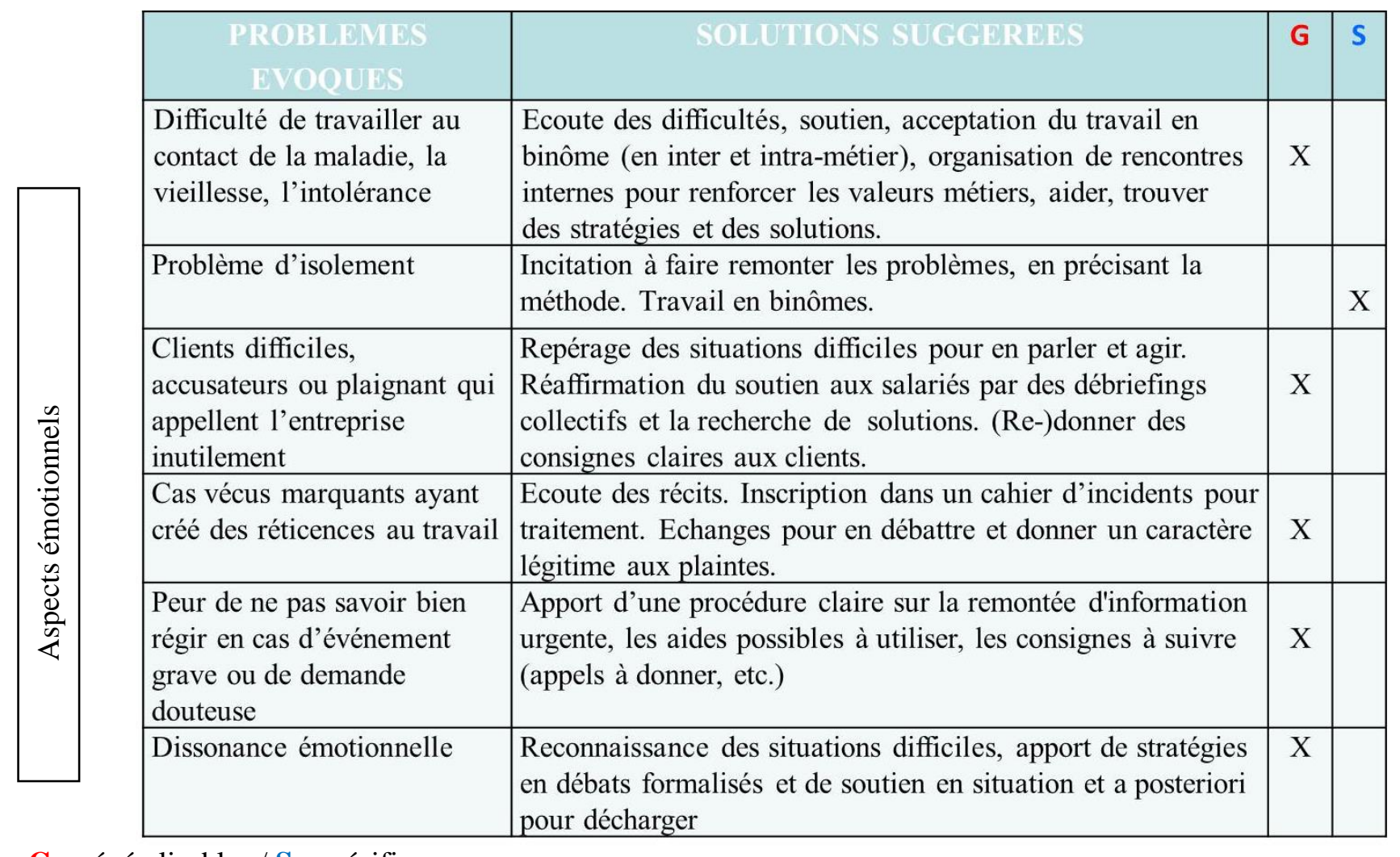

G : généralisables / S : spécifiques

Les actions avancées ou déjà en place dans l'entreprise visent à renforcer le dialogue interne, le travail collectif, la remontée d'informations, l'apport de solutions concrètes aux problèmes rencontrés, le soutien mutuel, l'entraide dans le travail, la confiance réciproque, le partage de représentations, le partage de valeurs professionnelles et la mise en œuvre des actions. Le but est d'améliorer :

- le savoir agir, qui dépend des savoirs (théoriques, pratiques, etc.), de l'expérience ;

- le vouloir agir, qui dépend de l'engagement, la motivation ;

- le pouvoir agir, qui dépend de l'organisation, des conditions de travail, et qui rend possible et légitime les prises de décision, d'initiative, de responsabilités et les actions.

\section{Discussion et conclusion}

L'étude a permis de confirmer les hypothèses émises.

La première hypothèse portait sur les situations à forte charge émotionnelle, créant non seulement des exigences émotionnelles élevées, mais aussi des effets négatifs à terme sur la santé et la motivation des salariés. L'étude a montré que la charge émotionnelle des intervenants à domicile comportait de nombreuses sources, accroissant l'ensemble des exigences émotionnelles, non négligeables pour la santé. La charge émotionnelle provient autant de la nature du travail que de la prescription. L'étude a confirmé que les salariés du secteur tentaient de gérer seuls la charge émotionnelle qu'ils considèrent comme partie intégrante du métier (Ribert-Van De Weerdt \& Baratta, 2016; Loriol, 2012). En cela, les exigences se trouvent augmentées. L'étude a montré aussi que la charge émotionnelle provenait des aspects relationnels du travail (le caractère agréable des clients, par exemple) mais aussi de problèmes de redéfinitions portant sur des aspects plus larges que les seuls aspects relationnels (par exemple, l'attribution de prestations sans rapport avec les qualifications) (Figure 3). Ceci confirme les travaux qui considèrent ces deux origines de la charge (Cahour, 2010, Cahour \& Lancry, 2011), et conforte l'hypothèse selon laquelle la charge émotionnelle augmente les exigences émotionnelles avec un retentissement sur la santé. Les résultats ont montré en effet des impacts sur la santé en termes de stress, 
souffrance au travail, malaises physiques, sentiment d'être vidé, etc.

La deuxième hypothèse a été également confirmée. Elle avançait le fait que la charge émotionnelle spécifique, relative au travail situé, pouvait avoir des effets à plus long terme sur la charge émotionnelle globale, se traduisant par le renforcement de l'engagement au travail (ou au contraire, le désengagement) et par une santé préservée (ou au contraire, des effets délétères). Les résultats de l'étude ont mis en évidence un impact, en montrant que l'accumulation de situations vécues à valence négative alourdissait la charge globale. Les exigences du travail pour les salariés à domicile sont constituées d'un ensemble cumulé de déterminants techniques et organisationnels, dont la part émotionnelle est importante. Les exigences émotionnelles créent une charge émotionnelle, qui, si elle n'est pas régulée de façon fonctionnelle dans des conditions optimales, peuvent devenir trop fortes et engendrer à terme des problèmes de santé. La définition de la charge émotionnelle spécifique que nous avions proposée est donc vérifiée pour ce secteur (Cahour \& Van De Weerdt, 2016 ; RibertVan De Weerdt \& Baratta, 2016).

La troisième hypothèse, qui concernait la possibilité de faciliter la régulation de l'activité et des émotions des salariés, exposés à des situations à forte charge émotionnelle, ou à des situations à charge émotionnelle plus modérée mais de façon répétée, a été vérifiée. La mise à disposition et le renforcement des ressources individuelles, collectives et organisationnelles a permis aux individus et collectifs de réguler la charge émotionnelle pour la maintenir à un niveau acceptable, et agir plus directement sur le travail et ses déterminants.

Sur le plan méthodologique, la recherche-intervention a montré que l'articulation de méthodes complémentaires comportait des bénéfices : les chroniques d'activité permettent de capturer l'espace-temps du travail, les entretiens et l'analyse des corpus amènent un vivier sur le vécu des salariés et leurs représentations personnelles du travail, les groupes de discussion (en séance d'auto-confrontation) fournissent une coconstruction sur l'objet du travail et une mise en débat du sens. C'est la combinaison de ces différents types de données récoltées qui conduisent à une mise en évidence des processus de régulation (de l'activité et des émotions) et une mise en débat du travail. La réitération de l'application de cette méthodologie permettra de savoir si cette mise en débat peut s'avérer pérenne dans la structure.

$\mathrm{Au}$ niveau empirique, nous sommes optimistes sur le fait que le plan devrait permettre : de faire diminuer les contraintes de travail, d'agir sur la réduction des exigences émotionnelles (par une meilleure préparation des missions des intervenants et une meilleure communication entre salariés sur les situations à forte charge émotionnelle susceptibles d'être rencontrées dans le travail quotidien), et de participer à la transformation du travail. Mais ce résultat reste à confirmer par une évaluation à plus long terme.

Quant aux limites de l'intervention, elles concernent premièrement la représentativité. Même si la recherche-intervention a été menée en profondeur (avec la participation de quasiment l'ensemble du personnel) et de façon longitudinale (sur une durée de 18 mois), une seule entreprise a été explorée. Deuxièmement, la démarche présente la particularité d'être « lourde » à utiliser (en raison de la présence des caméras et des autoconfrontations collectives qui nécessitent du temps de traitement des séquences). Par ailleurs, le fait d'avoir accès aux émotions par des méthodes exclusivement verbales fait appel aux émotions conscientisables, ce qui limite le repérage des émotions qui resteraient difficiles d'accès à la conscience. Mais cette méthode d'approche des émotions présente néanmoins l'intérêt de garantir a minima que les émotions exprimées par la personne sont bien en lien avec son travail, cette personne étant la mieux à même de faire ce lien sur la base de son vécu subjectif.

En ce qui concerne les perspectives, elles visent la construction d'une démarche de prévention positive visant à renforcer la motivation, le sens du travail et la santé des salariés, mais aussi à inciter la mobilisation et l'engagement des parties intéressées.

\section{Références Bibliographiques}

Ajzen, J. \& Kruglanski, A. (1983). Bias and error in human judgement. European Journal of Social Psychology, 13, 1, 1-44. Ashforth, B. \& Humphrey, R. (1993). Emotional Labor in Service Roles: The Influence of Identity. Academy of Management Executive, 18, 1, 88-115.

Baillieul, Y., Chaillot, G. \& Benoteau, I. (2013). Les services à la personne. Davantage sollicités dans les zones rurales et âgées. Insee première, 1461.

Briner, R.B. (1999). The neglect and importance of emotion at work. European journal of work and organizational psychology, 8, 3, 323-346.

Cahill, L. Haier, R.J, Fallon, J., Alkire, M.T., Tang, C., Keator, D., Wu, J., Mcgaugh, J.L. (1996). Amygdala activity at encoding correlated with long-term, free recall of emotional information. Proceedings of the national academy of sciences, 
93, 15, 8016-8021.

Cahour, B. (2010). Emotions, affects et confort comme nouveaux déterminants de l'activité et de l'usage. In G. Valléry, \& M. Zouinar, M C. Leport (Eds), Ergonomie, conception de produits et services médiatisés. PUF : Paris.

Cahour, B. \& Lancry A. (2011). Émotions et activités professionnelles et quotidiennes. Le travail humain, 74, 2, 97-106.

Cahour, B. \& Van De Weerdt, C. (2016). Emotions au travail : définition, processus et approches méthodologiques. In M.E. Bobillier-Chaumon, E. Brangier, M. Dubois, G. Vallery (Eds.), Psychologie du travail et des organisations : 100 notions clés. Recensement des notions en psychologie du travail et des organisations. Paris : Dunod, pp.164-168.

Cloutier, E., David, H. \& Teiger, C. (2003). Agir sur les conditions de travail des auxiliaires de vie : croiser les approches. Travail et emploi, 94, 75-83.

Cloutier, E., David Hélène, Teiger Catherine \& Prévost J. (1999). Les compétences des auxiliaires familiales et sociales expérimentées dans la gestion des contraintes de temps et des risques de santé. Formation Emploi, 67, 63-75.

Cosnier, J. (1994). Psychologie des émotions et des sentiments. Paris : Retz.

Cox, T. (1993). Stress research and stress management: Putting theory to work. HSE Contract Research Report 61/1993. Sudbury: HSE Books.

Cox, T., Griffiths, A. \& Leka, S. (2005). Work organization and work-related stress. In K. Gardiner \& J.M. Harrington

Eds.), Occupational Hygiene (3rd ed.). Oxford: Blackwell Publishing.

Damasio, A.R. (1994). L'erreur de Descartes : la raison des émotions. Paris, Ed. Odile Jacob.

Damasio, A.R. (1999). The feeling of what happens: body and emotion in the making of consciousness. New York: Harcourt brace.

Dares (2014). Les conditions de travail des salariés dans le secteur privé et la fonction publique. Dares Analyses, décembre 102.

Dares (2015). Les services à la personne en 2013. Un fort recul de l'emploi direct accentue la baisse de l'activité du secteur. Dares analyses, 11.

David, H., Cloutier, E., Teiger, C. \& Prévost, J. (2000). Réflexions sur une expérience interdisciplinaire dans le cadre d'une recherche exploratoire. Perspectives interdisciplinaires sur le travail et la santé [En ligne], 2-1, 2000.

Eurofound (2016). Sixth European Working Conditions Survey - Overview report. Publications Office of the European Union, Luxembourg.

Faucheux, C. \& Moscovici, S. (1971). Psychologie sociale théorique et expérimentale. Paris - la Haye : Mouton Ed.

Gagnon, I., David, H., Cloutier, E., Ouellet, F., Ledoux, E., Bourdouxhe, M. \& Teiger, C. (2003). Organisation du travail et développement de stratégies protectrices : cas d'auxiliaires sociales et familiales de services publics de maintien à domicile. Gérontologie et société, 4, 26, 107, 131-148.

Garabige, A. (2015). L'aide à domicile : un métier à l'épreuve des contraintes financières. Centre d'études de l'emploi, Connaissance de l'emploi, 123, 1-4.

Gollac, M. \& Bodier, M. (2010). Mesurer les facteurs psychosociaux de risques au travail pour les maîtriser. Rapport du collège d'expertise sur le suivi des risques psychosociaux au travail, faisant suite à la demande du ministre du Travail, de L'emploi et de la Santé, Paris, 223.

Grosjean, V. \& Ribert-Van De Weerdt, C. (2005). Vers une psychologie ergonomique du bien-être et des émotions : les effets du contrôle dans les centres d'appels. Le travail humain, 69, 1, 355-378.

Grosjean, V. \& Ribert-Van De Weerdt, C. (2010). Travail émotionnel et identité dans la relation de service : le cas particulier des centres d'appels téléphoniques. In M.E. Bobiller-Chaumon, M. Dubois, \& D. Retour (Dir.), La relation de service : nouveaux usages, nouveaux acteurs. Bruxelles, Editions de Boeck Université, 101-122.

Hellemans, C. (2014). Charge émotionnelle. In P. P. Zawieja, \& F. Guarnieri (Eds.), Dictionnaire des risques psychosociaux, Paris: Seuil, 90-92.

Hochschild, A. R. (1983). The managed heart: Commercialization of human feeling. Berkeley: University of California Press.

Isen, A.M. (1993). Positive affect and decision making. In M. Lewis, \& J.M. Haviland (eds.), Handbook of emotions (pp.312-329). New York: Guilford.

Izard, C. (1977). Basic Emotions, Relations Among Emotions, and Emotion-Cognition Relations. Psychological Review, 1992, 99, 3, 561-565.

Klein, T. \& Long, K. (2013). Conditions de travail, organisation du travail et usages des TIC selon les métiers. Une exploitation de l'enquête Conditions de travail. Document de travail du Centre d'analyse systémique, 3.

Krauth-Gruber, S. (2009). La régulation des émotions. Revue électronique de Psychologie Sociale, 4, 32-39.

Lachance, R. (2006). Charge de travail mentale et surcharge: des concepts multidimensionnels et interactifs. Rapport du Département des fondements et pratiques en éducation Université Laval. 2006.

Ledoux, J.E. (1994). Ccognitive-emotional interactions in the brain. In p. Ekman, \& R.J. Davidson (eds.), The nature of emotion: fundamental questions (pp. 216-223). New York: oxford university press.

Leduc, S. \& Valléry, G. (2006). Approche des compétences sociales par l'analyse de l'activité : une étude chez les aides à domicile. L'orientation scolaire et professionnelle, 35, 3, 387-419.

Lerner, J. S. \& Tiedens, L. Z. (2006). Portrait of the angry decision maker: How appraisal tendencies shape anger's 
influence on cognition. Journal of Behavioral Decision Making, 19, 115-137.

Loriol, M. (2012). La construction du social. Souffrance, travail et catégorisation des usagers dans l'action publique. Rennes : Presses Universitaires de Rennes.

Mann, S. (2004). 'People-work': emotion management, stress and coping. British Journal of Guidance \& Counselling, 32, 2, 205-221.

Ribert-Van De Weerdt, C. \& Baratta, R. (2016). L'analyse de l'activité et des émotions : regard sur une méthode en évolution à partir d'une étude de cas. Le travail humain, 1, 79, 31-52.

Ribert-Van de Weerdt, C. (2011). Les contraintes de travail et les stratégies de régulation émotionnelle en centre de relation clientèle, Le Travail Humain, 74, 4, 321-339.

Rimé, B. (2005). Le partage social des émotions. Paris : Presses Universitaires de France.

Rocher, M., Langevin, L., Castejon, C., Frachon, M., Poete, V. \& Villatte, R. (2005). Regard sur le travail : quand les aides à domicile deviennent "auxiliaires de vie sociale" pour agir sur leurs conditions de travail et sur leur santé. Note Scientifique et Technique de l'INRS, NS 257.

Scherer, K. (2006). Le poids de l'émotion. Entretien. Sciences humaines, 171, 42-43.

Soares, A. (2002). Le prix d'un sourire. Travail, émotion et santé dans les services. In D. Harisson et C. Legendre (Eds.), Santé, sécurité et transformation du travail. Presses de l’Université du Québec, 229-250.

Totterdell, P. \& Holman, D. (2003). Emotion regulation in customer service roles: Testing a model of emotional labor. Journal of Occupational Health Psychology, 8, 55-73.

Tversky, A. \& Kahneman, D. (1981). The Framing of Decisions and the Psychology of Choice. Science, New Series, 211, 4481, 453-458.

Tversky, A. \& Kahneman, D. (1974). Judgment under Uncertainty: Heuristics and Biases. Science, New Series, 185, 4157, 1124-1131.

Valléry, G. \& Leduc, S. (2010). Une analyse des emplois de services à destination des personnes âgées : compétences sociales et identité professionnelle des aides à domicile. Gérontologie et société, 135, 213-237.

Van De Weerdt, C. (2011). Conditions de travail, activité et stratégies émotionnelles de soignants à domicile. In C. Bastien, J. Cegarra, A. Chevalier, J. Dinet, N. Gregori \& V. Grosjean (Eds.), Epique'2011. Sixième Colloque de Psychologie Ergonomique (pp. 297-303). Nancy: Presses Universitaires de Nancy.

Zapf, D. (2008). The Psychology of Service: Interacting with Customers and Clients. Communication présentée le 12 mars 2008, Université de Francfort. 